Suppressing Electron Turbulence and Triggering Internal Transport Barriers with Reversed Magnetic Shear in the National Spherical Torus Experiment

J. L. Peterson, R. Bell, J. Candy, W. Guttenfelder, G. W. Hammett, S. M. Kaye, B. LeBlanc, D. R. Mikkelsen, D. R. Smith, H. Y. Yuh

December 8, 2011

Physics of Plasmas 
This document was prepared as an account of work sponsored by an agency of the United States government. Neither the United States government nor Lawrence Livermore National Security, LLC, nor any of their employees makes any warranty, expressed or implied, or assumes any legal liability or responsibility for the accuracy, completeness, or usefulness of any information, apparatus, product, or process disclosed, or represents that its use would not infringe privately owned rights. Reference herein to any specific commercial product, process, or service by trade name, trademark, manufacturer, or otherwise does not necessarily constitute or imply its endorsement, recommendation, or favoring by the United States government or Lawrence Livermore National Security, LLC. The views and opinions of authors expressed herein do not necessarily state or reflect those of the United States government or Lawrence Livermore National Security, LLC, and shall not be used for advertising or product endorsement purposes. 


\title{
Suppressing Electron Turbulence and Triggering Internal Transport Barriers with Reversed Magnetic Shear in the National Spherical Torus Experiment
}

\author{
J. L. Peterson, ${ }^{1}$ R. Bell, ${ }^{2}$ J. Candy, ${ }^{3}$ W. Guttenfelder, ${ }^{2}$ G. W. Hammett, ${ }^{2}$ S. M. Kaye, ${ }^{2}$ \\ B. LeBlanc, ${ }^{2}$ D. R. Mikkelsen, ${ }^{2}$ D. R. Smith, ${ }^{4}$ and H. Y. Yuh ${ }^{5}$ \\ 1) Lawrence Livermore National Laboratory, Livermore, California 94550, \\ $U S A$ \\ 2) Princeton Plasma Physics Laboratory, Princeton University, Princeton, \\ New Jersey 08543, USA \\ 3) General Atomics, San Diego, California 92186, USA \\ 4) Department of Engineering Physics, University of Wisconsin-Madison, Madison, \\ Wisconsin 53706, USA \\ 5) Nova Photonics Inc., Princeton, New Jersey 08540, USA
}

(Dated: 12 April 2012)

The National Spherical Torus Experiment (NSTX) [M. Ono et al., Nucl. Fusion 40, 557 (2000)] can achieve high electron plasma confinement regimes that are supercritically unstable to the electron temperature gradient driven (ETG) instability. These plasmas, dubbed electron internal transport barriers (e-ITBs), occur when the magnetic shear becomes strongly negative. Using the gyrokinetic code GYRO [J. Candy and R. E. Waltz, J. Comp. Phys. 186, 545 (2003)], the first nonlinear ETG simulations of NSTX e-ITB plasmas reinforce this observation. Local simulations identify a strongly upshifted nonlinear critical gradient for thermal transport that depends on magnetic shear. Global simulations show e-ITB formation can occur when the magnetic shear becomes strongly negative. While the ETG-driven thermal flux at the outer edge of the barrier is large enough to be experimentally relevant, the turbulence cannot propagate past the barrier into the plasma interior.

PACS numbers: 52.25.Fi, 52.35.Ra, 52.55.Fa, 52.65.Tt

Keywords: plasma turbulence, plasma simulation, plasma kinetic theory, plasma toroidal confinement 


\section{INTRODUCTION}

One of the challenges facing the development of magnetic confinement fusion energy is understanding and controlling turbulent transport. The National Spherical Torus Experiment $(\mathrm{NSTX})^{1}$ is aptly suited to studying electron transport in fusion plasmas. In many cases, electron thermal flux is the experiment's dominant loss mechanism ${ }^{2,3}$.

One possible source of electron thermal transport in NSTX is turbulence driven by the electron temperature gradient (ETG). The gyrokinetic ETG mode becomes unstable when plasma electron temperature gradients exceed a threshold value. A formula for this threshold for linear instability has been found for large-aspect ratio tokamaks ${ }^{4}$ :

$$
\left(\frac{R}{L_{T_{e}}}\right)_{\text {crit }}=\max \left\{(1+\tau)\left(1.33+1.91 \frac{\hat{s}}{q}\right)(1-1.5 \epsilon)\left(1+0.3 \epsilon \frac{d \kappa}{d \epsilon}\right), 0.8 \frac{R}{L_{n_{e}}}\right\} .
$$

Key factors in Eq. 1 include the plasma's major radius $R$, minor radius $r$, inverse aspect ratio $\epsilon$, elongation $\kappa$, safety factor $q$, and magnetic shear $\hat{s} \doteq(r / q)(\partial q / \partial r)$. Gradients of electron temperature $T_{e}$ and density $n_{e}$ are defined in terms of their logarithmic derivative with respect to the minor radius: $L_{x} \doteq-x /(\partial x / \partial r)$. The final key piece affecting the linear instability of the ETG mode is the parameter $\tau$ :

$$
\tau=Z_{e f f} \frac{T_{e}}{T_{i}} .
$$

While only calibrated to a finite range of parameters, in particular $\hat{s}>0$ and large-aspect ratio devices, Eq. 1 still yields some insight into the expected behavior of the ETG mode. Large electron temperature gradients drive the instability. Increasing $\tau$ stabilizes this drive. The safety factor and magnetic shear can also affect the linear ETG mode.

Significant thermal transport is possible from turbulence driven by the ETG mode ${ }^{5-13}$. The level of thermal transport depends largely on the magnetic shear. For cases with $\hat{s}>0$, ETG-driven turbulence can be large, as is the associated level of saturated thermal transport. But for $\hat{s}<0$, ETG-driven turbulent transport is much smaller.

Experiments on NSTX have found that when the ETG mode is unstable, a high-k collective scattering diagnostic ${ }^{14}$ observes enhanced electron-scale density fluctuations ${ }^{15,16}$. The fluctuations are consistent with the ETG mode, suggesting that when the electron temperature exceeds the requisite threshold, ETG driven turbulence appears in NSTX. These

plasmas' gradients cannot greatly exceed this linear threshold, sometimes hovering close to marginality. 
However, in some situations NSTX electron temperature gradients can greatly exceed the critical value for ETG instability. These plasmas are known to display electron internal transport barriers (e-ITBs) ${ }^{17,18}$. Interior to the barrier, electron temperatures can grow to very high values, while the local gradient at the e-ITB can exceed two to three times the ETG linear critical gradient. Additionally, high-k fluctuation activity generally decreases during an e-ITB discharge, even though the plasma is super-critically unstable to the ETG mode. e-ITBs can be formed with both radio-frequency $(\mathrm{RF})$ heating and neutral beam injection, in deuterium or helium. The one common link between all e-ITBs is negative magnetic shear, $\hat{s}<0$. No e-ITBs have ever been found with positive $\hat{s}$. Experimental evidence from NSTX suggests that reversing the magnetic shear reduces ETG-driven turbulent transport and triggers electron internal transport barriers.

The goal of this work is to explore these experimental observations with gyrokinetic simulation, focusing on a particular NSTX discharge, NSTX shot 129354, a deuterium RFheated e-ITB plasma. NBI beam blips were used for diagnostic purposes. Shown in Fig. 1 are profiles of the electron and ion temperature, the safety factor and the magnetic shear during the e-ITB phase at $232 \mathrm{~ms}$. The electron temperature, safety factor and magnetic shear of a non-e-ITB RF plasma (NSTX shot 124948 at $300 \mathrm{~ms}$ ) is included for reference. Although shot 124948 does have a $q_{\min }$ surface at $r / a \approx 0.4$, and therefore the magnetic shear is slightly negative, its electron temperature gradients are relatively uniform across the plasma. The e-ITB plasma shows increased electron temperature gradients relative to the non-e-ITB case from approximately $r / a=0.25$ to $r / a=0.39$, marking the boundaries of the transport barrier. The e-ITB's $q_{\min }$ and $\hat{s}_{\text {min }}$ are at approximately $r / a=0.42$ and $r / a=0.31$ respectively. For local simulations we will focus on a location near the center of the barrier, where local Miller equilibrium parameters ${ }^{19}$ include: $r / a=0.3, R / a=$ $1.55, \Delta^{\prime}=-0.27, \kappa=1.76, s_{\kappa}=-0.35, \delta=0.11, s_{\delta}=-0.073, \zeta=-2.0 \times 10^{-3}, s_{\zeta}=$ $0.13, q=2.40, \hat{s}=-2.40, \rho_{\star}=1.15 \times 10^{-2}$. The density gradient length at this time is given by $R / L_{n_{e}}=1.79$.

This work employs the gyrokinetic code GYRO ${ }^{20,21}$, beginning in Section II with an exploration of the linear instability properties of the reversed shear e-ITB discharge and continuing in Section III with the first local and non-local (global) nonlinear gyrokinetic simulations of ETG turbulence during an NSTX e-ITB. Conclusions appear in Section IV. 


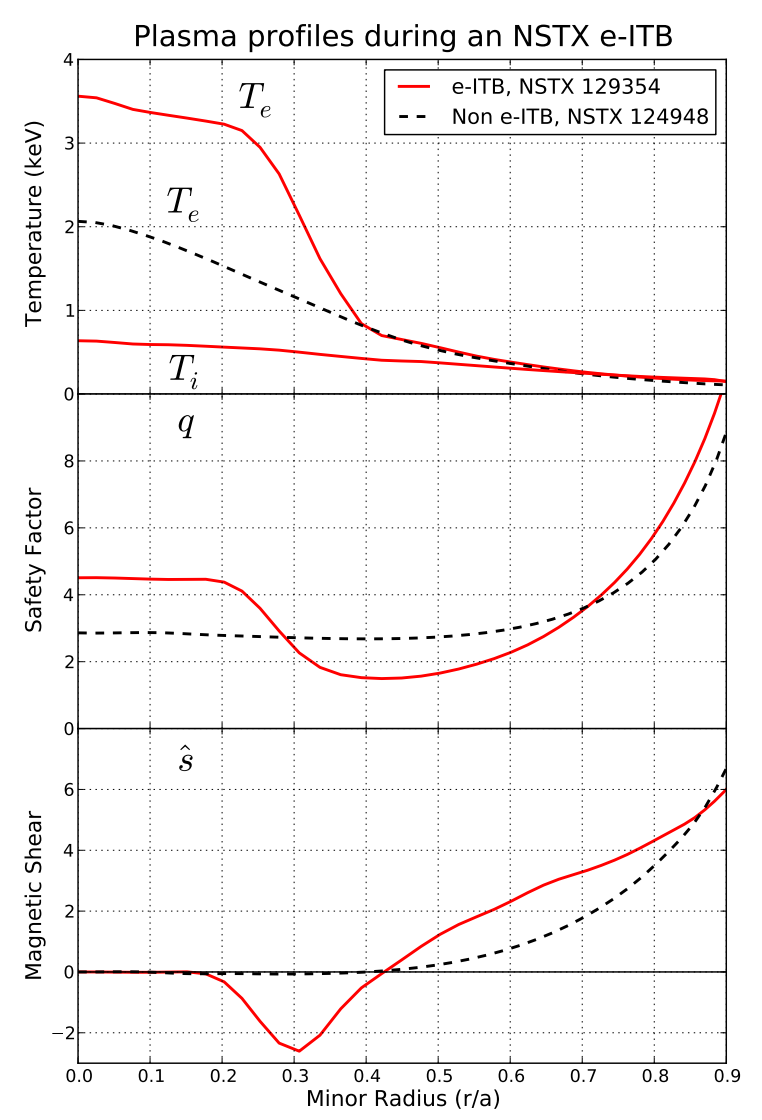

FIG. 1. Profiles of temperature, safety factor and magnetic shear for an RF-heated NSTX electron internal transport barrier (e-ITB), shot 129354 at $232 \mathrm{~ms}$ (solid). The electron temperature, safety factor and magnetic shear for a non- e-ITB RF NSTX plasma, shot 124948 at 300 ms, are shown for comparison (dashed).

\section{LINEAR ANALYSIS}

A possible explanation for the observed electron temperature gradients during an e-ITB is an increase in the linear threshold for ETG instability. That is, conditions in the plasma may be such that the ETG mode is stabilized up to very large gradients. To test this hypothesis, it is necessary to examine the linear instability of the ETG mode during this discharge, taking into account measurement uncertainties on quantities that may alter the ETG drive. 


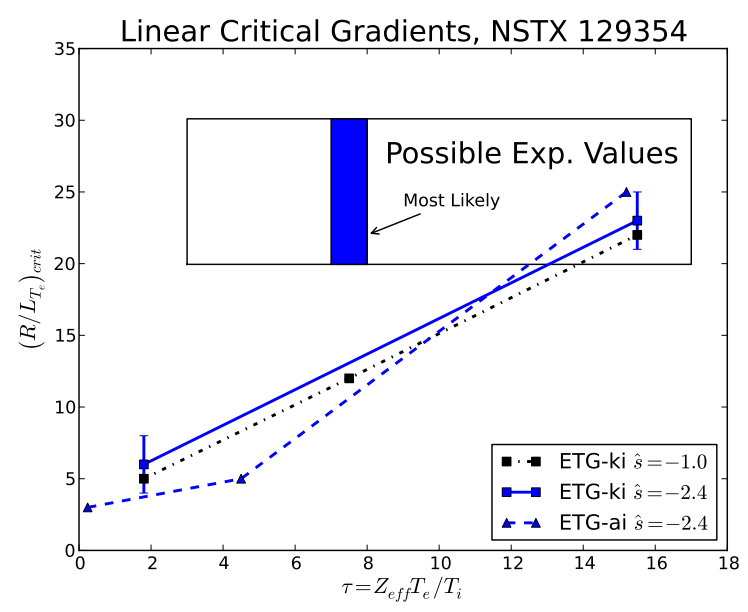

FIG. 2. Linear critical electron temperature gradients, for the parameters of NSTX 129354 during an e-ITB, testing the effects of $\tau, \hat{s}$ and ion model. ETG-ki uses gyrokinetic ions, and ETG-ai uses the adiabatic ion approximation. The boxes represent possible and mostly likely experimental values of $\tau$ and $R / L_{T_{e}}$ within the e-ITB, where $\hat{s}=-2.4$.

A major experimental uncertainty for the reversed shear e-ITB discharge stems from the impurity concentrations within the plasma. A best estimate of $Z_{\text {eff }}$ during this time is 2 , but experimental uncertainty allows for $Z_{\text {eff }}$ to lie between 1.5 and 3.5. As such, the value of $\tau$ within the barrier could vary from 3 to 17 , although the most likely value lies between 7 and 9. Figure 2 shows the effect of this uncertainty on the linear critical gradient for the ETG mode, $z_{c} \doteq\left(R / L_{T_{e}}\right)_{\text {crit }}$. Increasing $\tau$ increases $z_{c}$, as it does in Eq. 1 when the magnetic shear is positive, for simulations that use both the gyrokinetic ion (ETG-ki) and adiabatic ion (ETG-ai) models. Changing $\hat{s}$ from -1 to -2.4 uniformly raises $z_{c}$ by about 1 , making the effect due to $\tau$ the dominant driver of the ETG critical gradient. Fitting this trend yields an approximation of the effect of $\tau$ on the linear critical gradient:

$$
\left(\frac{R}{L_{T_{e}}}\right)_{\text {crit. }}=3.5+1.25 \tau .
$$

Both the coefficient on $\tau$ and its relative strength compared to the constant term are weaker in Eq. 3 than the $(1+\tau)$ dependence seen in Eq. 1 for $\hat{s} / q>0.5$. Figure 3 shows the observed gradients along with this estimate of the ETG critical gradient due to $\tau$ variation across the plasma profile, assuming $Z_{\text {eff }}=2$. The top and bottom of the gradient regions indicate values from $Z_{\text {eff }}$ of 3.5 and 1.5, respectively. Since Eq. 3 only considers variations 


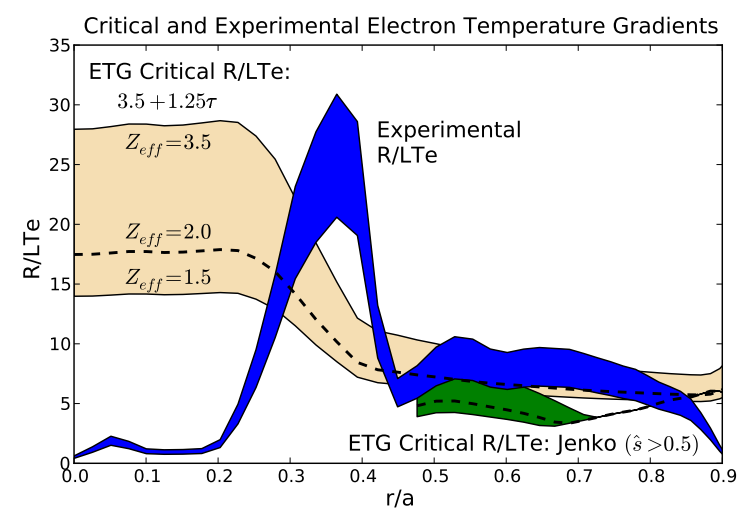

FIG. 3. Radial profiles of experimental and ETG linear critical gradients, NSTX 129354, using Eq. 3 to estimate the linear critical gradients. For reference, the critical gradient formula for positive shear large aspect ratio tokamaks ${ }^{4}$, Eq. 1 , is also plotted where $\hat{s}>0.5$. The lower bounds on the critical gradients represent $Z_{\text {eff }}=1.5$, the dashed profiles $Z_{e f f}=2$ and the upper bounds $Z_{\text {eff }}=3.5$.

in the critical gradient due to $\tau$ and ignores any geometrical dependence (such as an explicit $\epsilon$ dependence), the Jenko ${ }^{4}$ critical gradient formula, Eq. 1, is also plotted for reference to estimate geometrical effects at larger radii, where $\tau$ is no longer necessarily the dominant term. Since Eq. 1 was neither tested for spherical torii nor derived for negative magnetic shear, we only show it for $\hat{s}>0.5$. The plasma is divided into three regions. For $r / a>0.4$ the observed gradients and Eq. 3-calculated ETG critical gradients overlay. The Jenko formula gives a slightly lower critical gradient in the outer region than does Eq. 3, but both suggest that the ETG mode is at least marginally unstable outside of $r / a=0.4$. Between $r / a$ of 0.3 and 0.4 , the experimental values lie well above the ETG critical gradient, even considering the uncertainty due to $Z_{e f f}$. In this region, within the barrier, the plasma is very unstable to the ETG mode. However, the plasma in the interior, $r / a<0.3$, is stable to the ETG mode, making the mechanisms limiting the plasma gradients in this region unlikely to be ETG-driven flux. The present discussion focuses on the plasma in the barrier region with peak values of $R / L_{T_{e}}, 0.3<r / a<0.4$, which is very unstable to the ETG mode.

A scenario whereby observed e-ITB gradients can be explained by an increase in the linear critical gradient for the ETG mode due to increased impurity concentrations is unlikely for two reasons. Firstly, impurity concentrations would have to be very large, with $Z_{\text {eff }}$ 
approaching 3.5, to push the linear critical gradient large enough to account for observations. Secondly, the evolution of $Z_{\text {eff }}$ during the course of an e-ITB is not consistent with the plasma profile's evolution: $Z_{\text {eff }}$ typically either increases or stays constant when an e-ITB collapses. If the e-ITB's existence depended upon $Z_{\text {eff }}$ controlling the linear critical gradient, gradients should increase when $Z_{\text {eff }}$ rises, not decrease.

Take as a whole, this analysis suggests the existence of e-ITB plasmas is unlikely the result of linear ETG physics alone. Shot 129354 is super-critically unstable to the ETG mode within the barrier. The salient point is that this barrier exists, with measured experimental gradients well above the threshold for the linear ETG mode's instability. Near the barrier's base and outside of the barrier, the plasma may indeed be limited by the ETG mode, as profiles hover near marginality. But the linear ETG critical gradient cannot explain the observed e-ITB.

\section{NONLINEAR ANALYSIS}

Since linear ETG physics alone cannot explain the observed gradients, we turn now to nonlinear simulations of ETG turbulence to determine the level of ETG-driven thermal flux and the nonlinear critical gradient for transport, $z_{c}^{N L}$. We follow GYRO's notation ${ }^{21}$ and report in normalized ion units. Some key elements are: the device major radius, $R$; the full minor radius, $a$; the ion sound speed, $c_{s}=\sqrt{T_{e} / m_{D}}$; the ion sound radius, $\rho_{s}=c_{s}\left(m_{D} c / e B_{\text {unit }}\right)$; the electron thermal velocity, $v_{e}=\sqrt{T_{e} / m_{e}}$; the electron gyroradius, $\rho_{e}=v_{e}\left(m_{e} c / e B_{u n i t}\right)$; the gyroBohm unit diffusivity, $\chi_{G B, i}=\rho_{s}^{2} c_{s} / a$; and the gyroBohm energy flux, $Q_{G B, i}=$ $n_{e} c_{s} T_{e}\left(\rho_{s} / a\right)^{2} . n_{e / i}, T_{e / i}, m_{e / D}, c$ and $B_{\text {unit }}$ represent the background electron/deuterium density, temperature, the electron/deuterium mass, the speed of light and the effective magnetic field strength, respectively.

\section{A. Local Studies}

We begin with local flux tube simulations at $r / a=0.3$ to investigate the nonlinear ETG critical gradient $z_{c}^{N L}$ and how it relates to the linear critical gradient $z_{c}$.

Unless otherwise indicated, flux tube simulations follow 24 toroidal modes (up to a maximum $k_{\theta} \rho_{e}$ of $\left.1.004,\left(k_{\theta} \rho_{s}\right)_{\max }=60.25\right)$ with 256 radial grid points in a box with radial and 
binormal directions measuring $L_{x} \times L_{y}=4.26 \times 2.4 \rho_{s}=255.6 \times 144 \rho_{e}$. Radial convergence required $\Delta x<\rho_{e}$. Other resolutions are: $n_{\text {trap }}=44$ (the number of mesh points along a trapped particle orbit), $n_{b}=12$ (the number of parallel finite elements), $n_{\lambda}=12$ (the number of pitch angles for each sign of parallel velocity, split evenly between trapped and passing particles), $n_{\epsilon}=6$ (the number of energy grid points) and $\epsilon^{*}=6.0$ (the maximum simulated dimensionless energy). Temporal stability and convergence required time steps of $\Delta t\left(c_{s} / a\right)=3.3 \times 10^{-4}$, and simulation times of $t_{\max }\left(c_{s} / a\right)=10-50$. Simulations are electrostatic, without $\mathbf{E} \times \mathbf{B}$ shear, which is negligible for this RF-heated discharge. These parameters have been tested for convergence ${ }^{22}$ and agree with linear eigenmode resolution tests of NSTX plasmas ${ }^{23}$. These resolutions are very high, requiring expensive calculations: each flux tube simulation costs between 80,000 and 100,000 CPU-hours a piece on the ORNL Cray XT system.

\section{1. $\tau=7.5$}

We begin near the most likely value of $\tau=7.5$, simulating ions, electrons and carbon simultaneously: $n_{D} / n_{e}=0.867, n_{c} / n_{e}=0.022, T_{D} / T_{e}=T_{c} / T_{e}=0.22$, and $\hat{s}=-1$. The flux tube, however, remains electron-sized, with a default size of $L_{x} \times L_{y}=178 \times 140 \rho_{e}$, and $k_{\theta} \rho_{e}<1.03$. Figure 4 compares the electron heat fluxes as a function of driving gradient. The ETG-ki and ETG-ai models at the default box size agree fairly well up to very large gradients (although the ETG-ai runs consistently calculate less flux). The similarity between ETG-ki and ETG-ai runs shows that the ion response is nearly adiabatic, so the important parameter governing all ion behaviors is $\tau$ and not specific ion concentrations.

In general, Figure 4 indicates that ETG turbulent flux is very low, even at gradients well above the linear critical gradient for transport. Experimentally relevant levels of electron thermal flux are 0.15-0.2 in ion gyrobohm units. When driving the simulation with experimental gradients found in the e-ITB, corresponding to $R / L_{T_{e}}=20-30$, the ETG mode, despite being unstable, can only produce $Q_{e} / Q_{G B i}=0.02-0.04$. Even at driving gradients that are much higher than those seen in the experiment, the flux remains far below experimentally relevant levels.

Keeping in mind that typical experimental heat fluxes correspond to $Q_{e} \sim 0.15-0.2 Q_{G B i}$, in absolute units increasing the box size on the ETG-ki model has a modest effect on the 


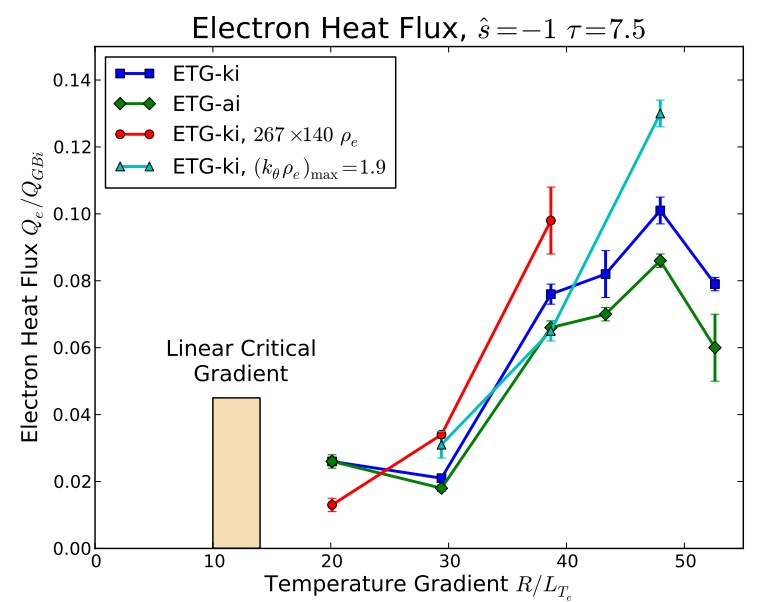

FIG. 4. Electron heat flux as a function of driving gradient for $\tau=7.5, \hat{s}=-1$. The experimental temperature gradient lengths and electron heat fluxes are respectively given by $R / L_{T_{e}}=20 \pm 5$ and $Q_{e} / Q_{G B i}=0.175 \pm 0.025$.

flux for $R / L_{T_{e}} \approx 40$, but less so at lower gradients. Including very high-k modes, up to $k_{\theta} \rho_{e}$ of 1.9 does increase the integrated flux at $R / L_{T_{e}} \approx 48$ by about $30 \%$ over the baseline case, but at more modest gradients the effect is negligible. This is due to the fact that the linear growth spectrum moves to higher-k as the temperature gradient increases; however, this is not the case below $R / L_{T_{e}} \approx 40$. While both the default box size and maximum mode number seem adequate in the range of experimentally relevant parameters, $R / L_{T_{e}} \lesssim 30$, neither increasing the box size nor including additional modes can push the calculated flux to experimentally relevant levels, even at gradients much larger than those observed in the experiment.

Flux tube simulations at $\tau=7.5$ show that electron transport remains low for driving gradients that are much higher than the linear critical gradient. Define the nonlinear critical gradient for significant turbulent transport as the electron temperature gradient above which the electron thermal flux exceeds experimentally relevant values, or $Q_{e} \geq 0.15 Q_{G B i}$. Because none of the simulations in Fig. 4 produce that level of transport, they collectively imply that the nonlinear critical gradient is given by $R / L_{T_{e}}>50$. This value rests well-above observations of e-ITB plasmas. One possible reason is that $\tau$ is too high, and the ETG drive is therefore too low. 


\section{2. $\tau=1.8$}

By neglecting carbon in the simulation and treating deuterium gyrokinetically with a mass-ratio $\mu_{e}=\sqrt{m_{D} / m_{e}}=60$, we reduce $\tau$ to 1.8 and increase the ETG drive. This corresponds to a plasma that is more unstable to the ETG mode, at parameters roughly consistent with plasma conditions at the foot of the e-ITB, $r / a \approx 0.4$. We also increase the energy grid resolution to $n_{\epsilon}=12$, although this changes the time-integrated thermal flux by less than 10 percent when compared to simulations at $n_{\epsilon}=6$, holding all other parameters fixed.

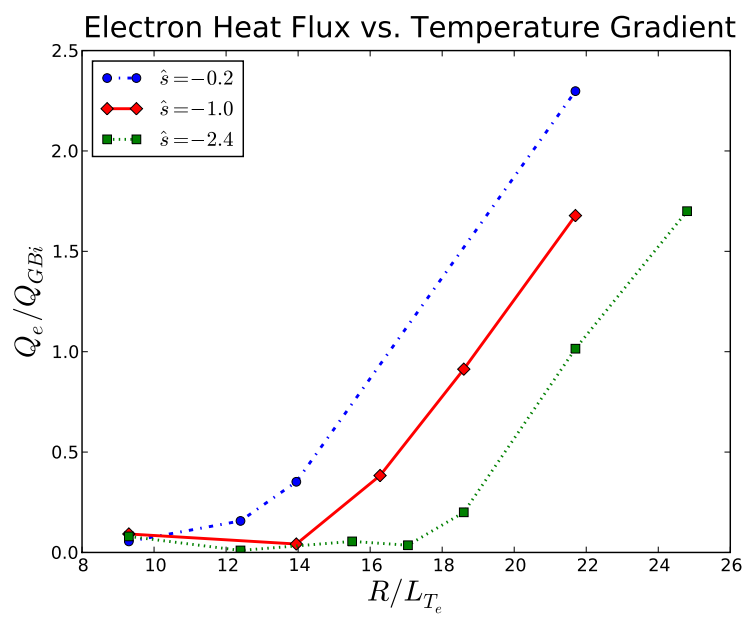

FIG. 5. Electron heat flux as a function of driving gradient and magnetic shear for $\tau=1.8$.

Primarily we find a large upshift in the critical gradient for transport, whose strength depends on magnetic shear. Figure 5 shows the electron heat fluxes for a number of driving gradients and magnetic shear. All simulations are linearly unstable to the ETG mode, but transport remains low below a threshold that increases as the magnetic shear becomes more negative. But, above that threshold, the turbulent flux increases and can approach and exceed experimentally inferred levels of $Q_{e} / Q_{G B i}=0.15-0.2$. This also corresponds to electron thermal diffusivities of roughly $1 \mathrm{~m}^{2} / \mathrm{s}$ at $R / L_{T_{e}}=15$. For concreteness, let the nonlinear critical gradient be given by $z_{c}^{N L}$, the driving inverse gradient length above which the electron thermal diffusivity from ETG-driven turbulence exceeds this level. Plotted in Fig. 6 is the dependence of the linear ETG critical gradient, $z_{c}$, the nonlinear critical gradient $z_{c}^{N L}$ and their difference, the magnitude of the nonlinear unshift, $\Delta z=z_{c}^{N L}-z_{c}$. For all 


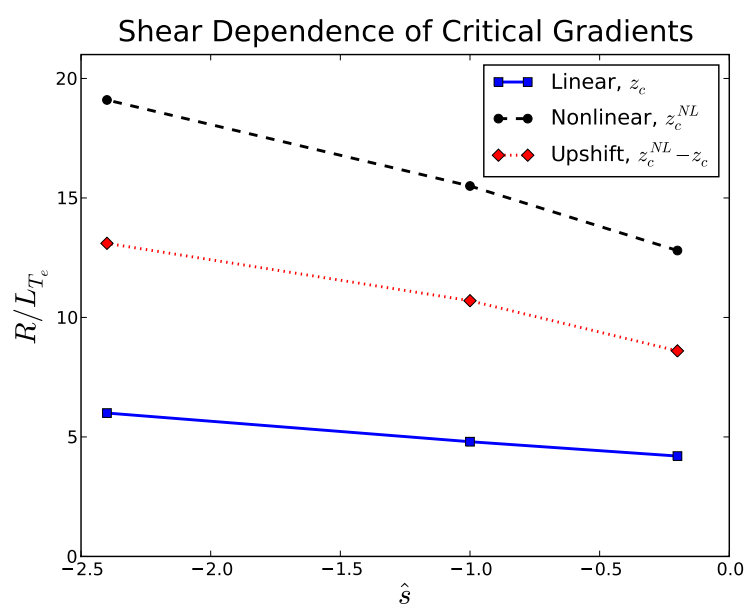

FIG. 6. Critical gradients as a function of magnetic shear, $\tau=1.8$. ETG becomes linearly unstable at gradients above $z_{c}$ (solid with square). Above $z_{c}^{N L}$ (dashed with circles), turbulent thermal diffusivities exceed $1 \mathrm{~m}^{2} / \mathrm{s}$. The dotted line with diamonds represents the nonlinear upshift of the critical gradient, $\Delta z=z_{c}^{N L}-z_{c}$. For comparison, the original cyclone ITG test case found an upshift in the critical gradient for transport that extended $R / L_{T_{i}}$ by 2 , from $z_{c}=4$ to $z_{c}^{N L}=6$ at $\hat{s}=0.78^{24}$.

tested values of shear, the upshift in the critical gradient is very strong, becoming stronger as the magnetic shear becomes more negative, ranging from 9 at $\hat{s}=-0.2$ to 13 at $\hat{s}=-2.4$.

This upshifted critical gradient for ETG transport is very large. As a comparison, the increase in the critical gradient for ITG transport due to zonal flows, known as the Dimits shift $^{24}$, represents an increase of the critical ITG gradient from $R / L_{T_{i}}=4$ to 6 for the Cyclone parameters. While the exact magnitude of the Dimits shift depends on specific problem parameters (the shift is reduced at higher $q$ or when including the effects of trapped electrons ${ }^{25}$, but can be enhanced by plasma shaping ${ }^{26}$ ), the shift in the nonlinear critical gradient for these reversed shear ETG simulations is relatively much stronger.

Additionally, the location of $z_{c}^{N L}$ is consistent with observed gradients during an e-ITB. $z_{c}^{N L}(\hat{s}=-2.4)=19$, which approaches the experimental range of 20-30. Although this value still lies below the experimental value, increasing $\tau$ to within the experimental range boosts $z_{c}^{N L}$, as demonstrated by the $\tau=7.5$ simulations of Section III A 1 .

Above $z_{c}^{N L}$, the nature of the turbulence can change. Some cases feature radially elongated streamers that lie not on the midplane, but at a finite poloidal elevation. Figure 7 

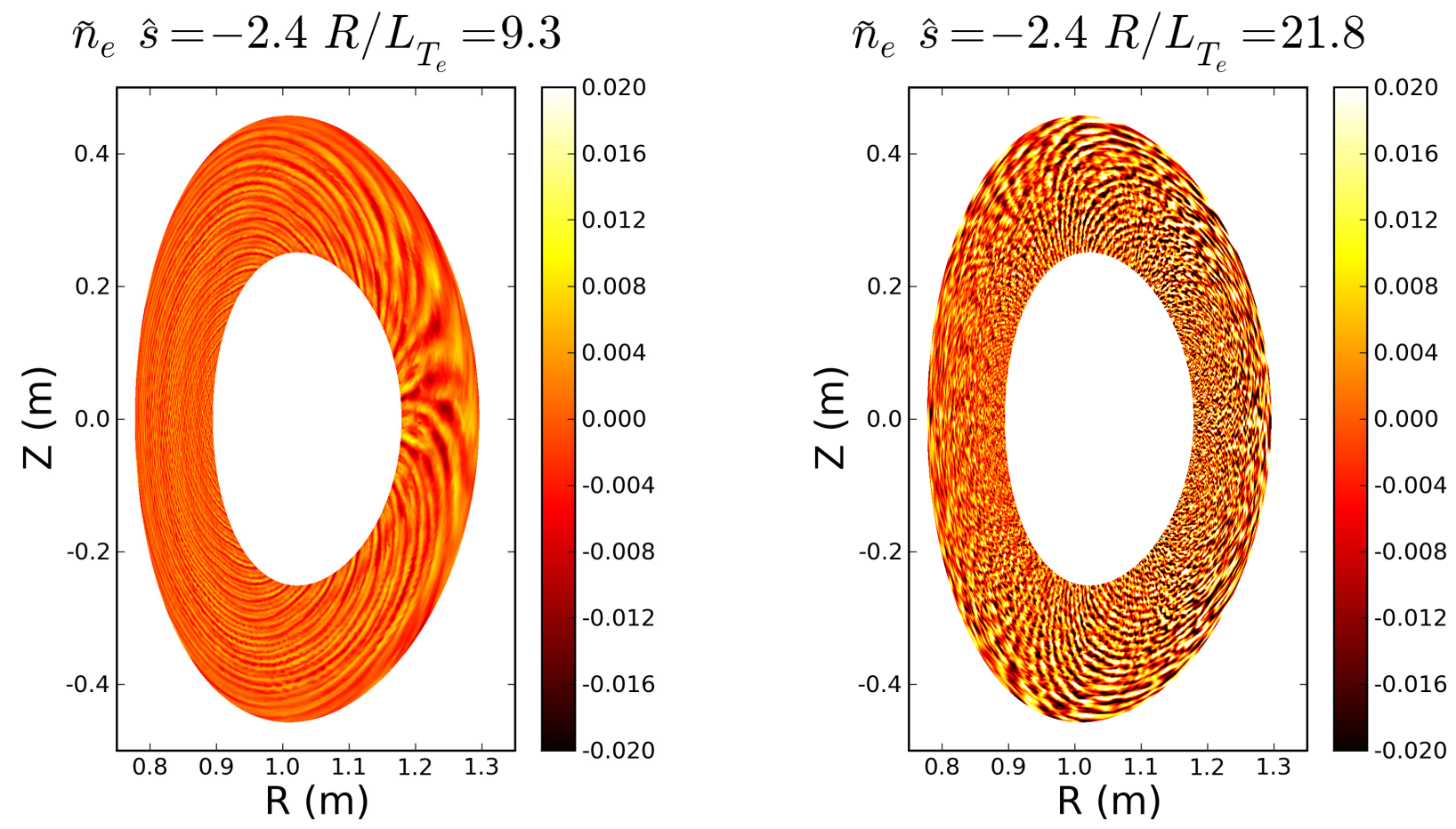

FIG. 7. Poloidal cross sections of saturated density fluctuations below and above $z_{c}^{N L}$ for $\hat{s}=-2.4$.

The flux-surface widths have been enhanced by a factor of four for visual clarity.

shows a poloidal cross section of density fluctuations for local simulations with $\hat{s}=-2.4$ at two different driving gradients. Below $z_{c}^{N L}$, at $R / L_{T_{e}}=9.3$ eddies rotate away from the midplane, consistent with theories ${ }^{27}$ of how reversed shear alters turbulence and reduces transport. Since radial eddies are rotated away from the magnetic axis, their effect on transport is mitigated. But above $z_{c}^{N L}$, the plasma fluctuations have a different character. The midplane is populated with small-scale broadband tubulence, while off the midplane, at poloidal angle $\theta= \pm \pi / 2$, large-scale structures appear. These elongated eddies point in the minor radial direction, which at these poloidal locations corresponds to aligning in the $Z$ direction, out of the top and bottom of the annulus.

These "off-midplane" streamers drive a significant amount of heat flux, accounting for roughly one quarter of the integrated total. They also are localized in a narrow toroidal spectral band. Figure 8 shows fluctuation powers as a function of poloidal angle and toroidal mode number. The strongest peak exists at $\theta \approx \pm \pi / 2$, the location of the off-midplane streamers. These peaks are not only very strong and poloidally localized, but also centered 


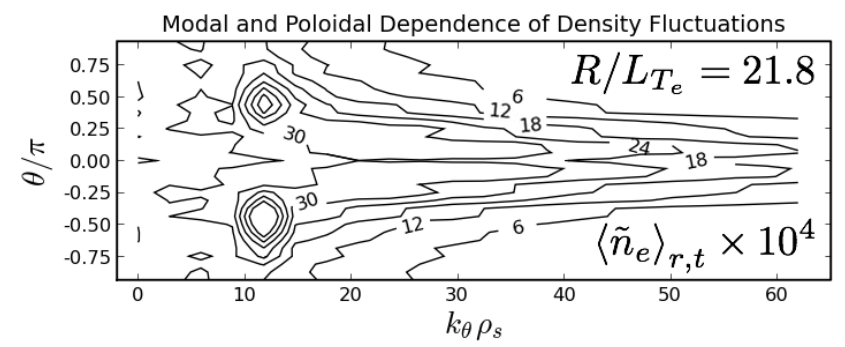

FIG. 8. Density fluctuations as a function of poloidal angle $\theta$ and $k_{\theta}$, showing off-midplane peaking at $k_{\theta} \rho_{s} \approx 13$.

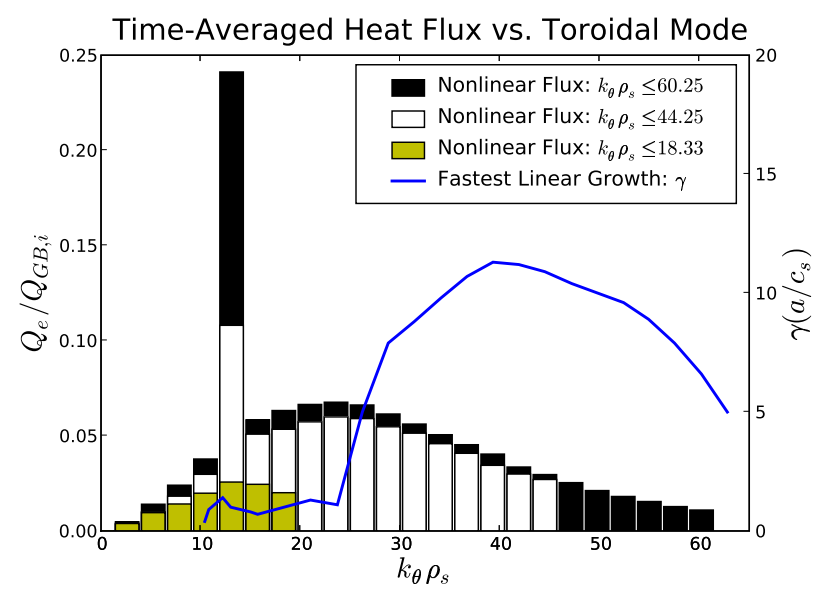

FIG. 9. Time averaged heat flux spectra for different values of $\left(k_{\theta} \rho_{s}\right)_{\max }, \hat{s}=-2.4, z=21.8$, and the spectrum of the fastest growing linear mode.

around $k_{\theta} \rho_{s}=13$. Furthermore, the midplane fluctuations exist in a broadband spectrum at higher mode numbers. Figure 9 shows the time-integrated heat flux spectra as a function of $k_{\theta} \rho_{s}$ from three different simulations, each with different values of the maximum $k_{\theta} \rho_{s}$. The heat-flux signatures of both the midplane broadband turbulence and the off-midplane streamers are visible, as is the linear growth rate spectrum. The turbulent flux has a broad spectrum and a sharp narrow peak, at the location of the off-midplane streamers, whose integrated flux $\left(0.25 Q_{G B, i}\right)$ is roughly one quarter of the total.

Furthermore, the off-midplane streamers may be nonlinearly driven. The linear growth rate spectrum in Fig. 9 has three main regions of instability: small peaks centered at $k_{\theta} \rho_{s}$ of 12 and 20, and a much larger one around 40. (Note that in this case the ETG modes are 
completely stable for $k_{\theta} \rho_{s}<10$.) While an instability exists near $k_{\theta} \rho_{s}=13$, failing to include the peak ETG drive at $k_{\theta} \rho_{s} \sim 40$ causes the streamers to disappear. A simulation with 48 modes (instead of 22) increases $\left(k_{\theta} \rho_{s}\right)_{\max }$ to 123.05 and contains all unstable ETG modes plus higher-k stable modes. In this case, the total heat flux increases by $\sim 35 \%$, in a manner consistent with the results of Fig. 4. This increase is largely due to the background spectrum centered about $k_{\theta} \rho_{s} \sim 20$, whose peak value increases to $\sim 0.1 Q_{G B, i}$, while the heat flux contribution from the streamers increases comparatively less, from 0.24 to $0.255 Q_{G B, i}$. Such behavior suggests that the ETG modes growing at $k_{\theta} \rho_{s} \sim 40$ are nonlinearly driving the off-midplane streamers.

In summation, the flux tube scans at $\tau=1.8$ have found not only a very strong nonlinear critical gradient, increased by reversed magnetic shear, but also off-midplane streamers, large-scale structures that are nonlinearly driven by ETG turbulence. However, their appearance is not ubiquitous. They only appear at very large gradients and strong reversed shear, and while this condition is necessary, it's not sufficient. In this sense, the streamers may or may not be important to this discharge, since the heat fluxes associated with them are many times the experimentally measured levels, and since they are found at values of $\tau$ lower than in the e-ITB. Yet, regardless of the applicability to this particular discharge, their existence is an interesting example of nonlinear turbulent interactions, energy cascades, secondary instabilities and coherent structures.

Taken as a whole, the first-of-a-kind flux tube simulations of NSTX e-ITBs suggest that their formation is caused by reversed magnetic shear, which suppresses ETG-driven thermal transport, thereby allowing the plasma to sustain gradients that are well above the linear threshold for instability. However, experimental uncertainties in $\tau$ limit the interpretation. Around an e-ITB, $\hat{s}$ and $T_{e}$ vary rapidly. Adjusting these parameters independently within a local flux tube simulation allows for the effects of each parameter to be isolated; however, it is their interaction that controls the performance of the experiment. To effectively capture this dynamic, one must allow for plasma profile variation. In other words, we proceed with global simulations of an electron internal transport barrier. 


\section{B. Global Studies}

To capture the structure of the e-ITB, the global ETG simulation domain includes most of the barrier and spans from $r / a=0.27$ to $r / a=0.43$, where $T_{e}$ drops from $\approx 2.7$ to $\approx 0.7$ $\mathrm{keV}$, corresponding to $T_{e} / T_{i} \approx 5.2$ at the inner radius and $\approx 1.7$ at the outer radius, as shown in Fig. 1. The goal will be to test two values of $Z_{\text {eff }}: 2.0$ and 3.5, which span the experimental range. $\tau$ for $Z_{\text {eff }}=2.0(3.5)$ ranges from $10.4-3.4(18.2-6.0)$. The main ion is deuterium, but an impurity carbon species is added to change $Z_{e f f}$, while satisfying quasineutrality.

With a realistic deuterium-electron mass ratio, $\mu_{e}=\sqrt{m_{D} / m_{e}}=60$, the domain is $862.2 \rho_{e}$ in width, requiring 864 radial grid points. To maximize the useful radial domain, and based on work ${ }^{28}$ exploring necessary buffer widths for ST ETG simulations, we chose a buffer that is 16 grid points wide, but employ strong electron damping, $\nu_{e}^{\text {buffer }}=60\left(c_{s} / a\right)$, and turn on the adaptive source. Furthermore, as the ETG-ki and ETG-ai models show little difference in the flux tube simulations of Fig. 4, ions are treated adiabatically. Again, the simulations are electrostatic and do not include $\mathbf{E} \times \mathbf{B}$ shearing. In total, each nonlocal simulation costs over 150,000 CPU-hours.

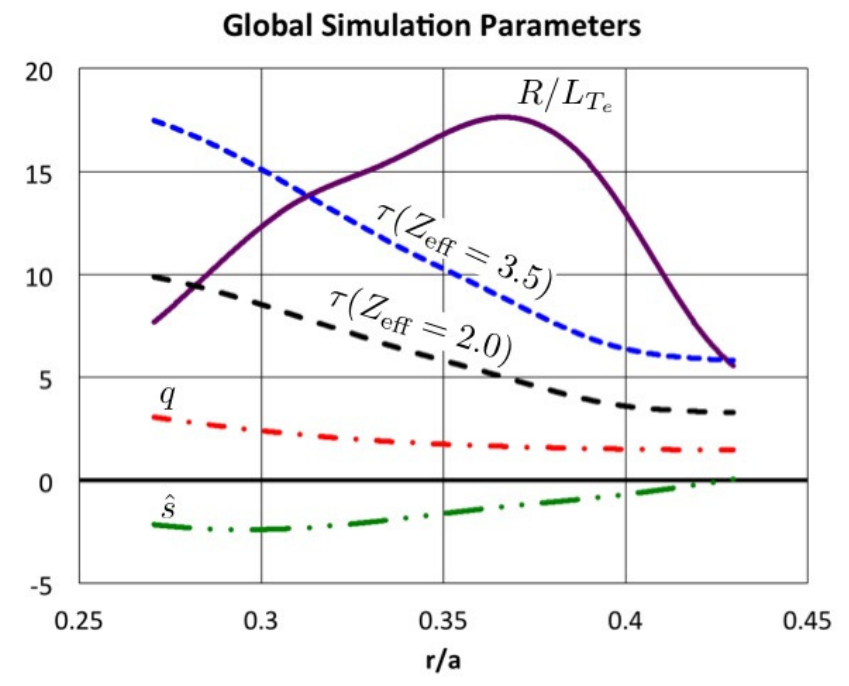

FIG. 10. Plasma parameters and simulation domain used in the NSTX 129354 global simulations.

Figure 10 shows the radial simulation domain and variation of $R / L_{T_{e}}, \tau$ (for the two different values of $Z_{\text {eff }}$ ), $q$ and $\hat{s}$. The minima of both $\hat{s}$ and $q$ are included in the simulation. 
The minimum value of $\hat{s}$, occurring at $r / a=0.296$, is -2.408 . The minimum value of $q$ is 1.466 , at $r / a=0.428$. Inside of this radial location $\hat{s}<0$. The largest electron temperature gradient occurs at $r / a=0.367$, where $\hat{s}=-1.28$. According to Eq. 3, the maximum value of $R / L_{T_{e}}-\left(R / L_{T_{e}}\right)_{\mathrm{ETG} \text { crit }}$ occurs at $r / a=0.374(0.380)$ for $Z_{e f f}=2.0(3.5)$.

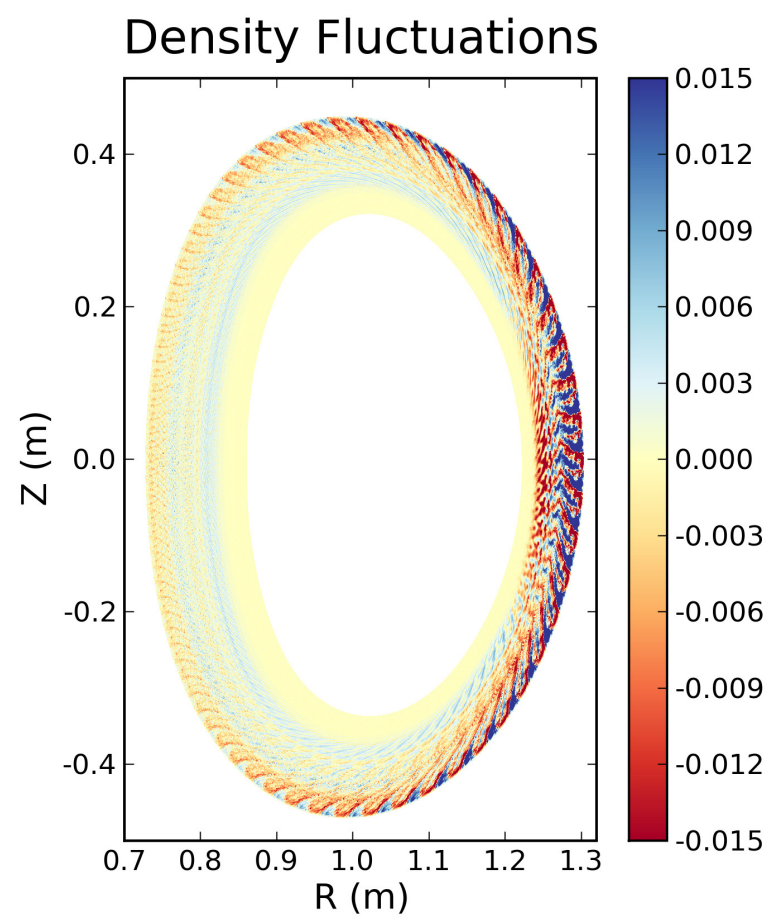

FIG. 11. Cross-section of electron density fluctuations, 129354 global simulation, showing an electron internal transport barrier. $Z_{e f f}=2.0 t=19.18\left(a / c_{s}\right)$. Although peak amplitudes of $\pm 5 \%$ exist, for clarity only $\tilde{n}_{e} \leq \pm 1.5 \%$ are shown.

The importance of reversed magnetic shear in barrier formation can be seen in Figure 11, a poloidal cross-section of density fluctuations for the simulation with $Z_{\text {eff }}=2$. ETGdriven turbulent eddies exist at outer radii, but the turbulence level drops at smaller radii and disappears entirely within $r / a \sim 0.3$, where $\tau$ is larger and $\hat{s}$ is more negative.

Though ETG turbulence exists in the simulation, its amplitude is greater at the outer radii, where $\hat{s}$ is the least negative, than at inner radii, where the temperature gradients are the largest. Figure 12 shows a time history of the electron heat flux as a function of minor radius. The location of minimum magnetic shear (most negative) is marked. The heat flux grows first outside of $\hat{s}_{\min }$ and later elsewhere, with some turbulent spreading. A 


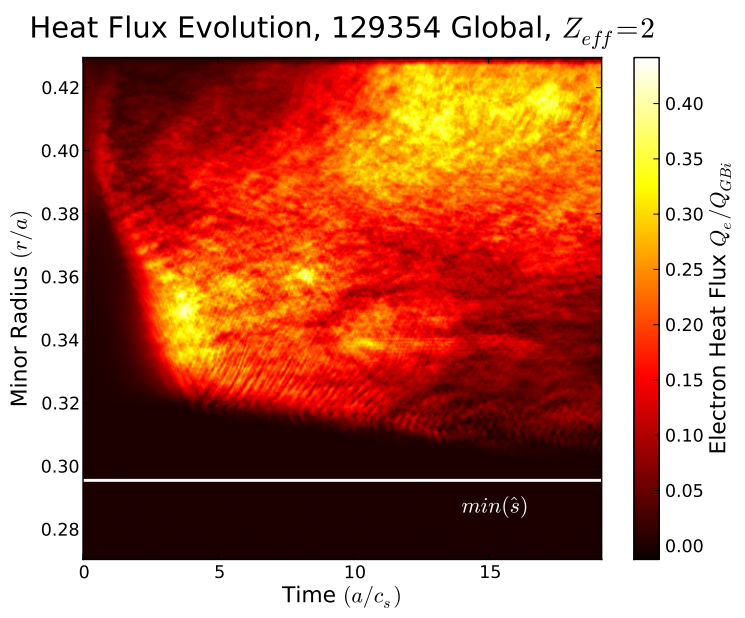

FIG. 12. Time evolution of radial heat flux profile, 129354 global simulation, $Z_{\text {eff }}=2$. The white line marks the location of minimum $\hat{s}$. Heat fluxes as calculated by TRANSP increase as one moves radially from $\sim 0.16 Q_{G B i},(r / a=0.35)$ to $0.19 Q_{G B i}(r / a=0.40)$.

transient burst at $r / a=0.35$ grows up and peaks near time $t=5\left(a / c_{s}\right)$ but dies at later times. In all, the flux never reaches significant levels inside $r / a \approx 0.31$ and by $t \approx 11\left(a / c_{s}\right)$ a steady-state appears, with large heat flux at large $r$ and negligible at small $r$. While some turbulence spreading may exist in part of the simulation, Fig. 12 illustrates that turbulent eddies are prevented from propagating into the deeper parts of the transport barrier, where the magnetic shear is strongly negative and $\tau$ is large. For a few reasons, the remaining turbulence seen in the outer part of the simulation is consistent with a reduction of transport within the barrier due to magnetic shear. Firstly, the peak flux approaches experimentally relevant levels of $\sim 0.15-0.2 Q_{G B, i}$, suggesting that this flux is strong enough to limit experimental gradients in the outer part of the barrier. Secondly, the peak electron flux occurs not where linear ETG drive is the largest, at $r / a=0.37$, but where the magnetic shear is least negative. This is consistent with the local simulations of Sec. III A that showed simulations with more negative magnetic shear producing lower levels of transport for a given driving gradient. In other words, because of the reduction of turbulence in a transport barrier, the gradients must be much steeper to drive sufficient heat flux to balance heat sources. In the inner part of the simulation, where ETG turbulence is found to be completely suppressed, residual transport from some other mechanism presumably limits temperature gradients. 


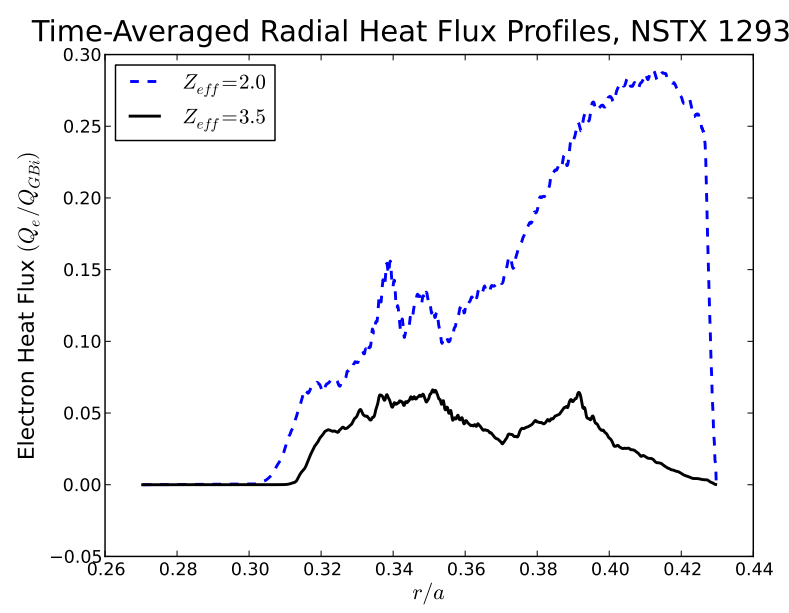

FIG. 13. Time-averaged heat flux profiles for global simulations with different values of $Z_{\text {eff }}$. For reference, experimentally inferred levels range from $0.16 Q_{G B i},(r / a=0.35)$ to $0.19 Q_{G B i}(r / a=$ $0.40)$.

Altering $Z_{\text {eff }}$ does not qualitatively change the existence of the electron internal transport barrier. The radial heat flux profiles of the two simulations, shown in Fig. 13, follow similar patterns. Overall, lowering $Z_{\text {eff }}$ increases the transport, which is to be expected, since it coincides with stronger ETG drive, but both profiles show negligible heat flux inside $r / a=0.3$. At larger radii, however, ETG-driven flux can be experimentally relevant, which was calculated by TRANSP to range from $\sim 0.16 Q_{G B i}(r / a=0.35)$ to $0.19 Q_{G B i}(r / a=0.40)$. The $Z_{\text {eff }}=3.5$ case seems to under-predict experimental levels of heat flux. While such a high value of $Z_{\text {eff }}$ cannot be ruled out a priori, the most likely experimental value is closer to 2. At this value of $Z_{\text {eff }}$, ETG can drive enough flux to account for experimentally measured levels in the outer parts of the transport barrier. But, the key point is that both profiles show the existence of a transport barrier and that turbulence is suppressed in the inner parts of the barrier, as the magnetic shear becomes more negative and $\tau$ gets larger.

\section{CONCLUSIONS}

In summation, NSTX can reach high electron confinement modes that display electron internal transport barriers. These plasmas have large negative values of magnetic shear and can support electron temperature gradients that are super-critically unstable to the ETG 
mode.

The first nonlinear gyrokinetic simulations of an NSTX e-ITB support the experimental observations of reversed magnetic shear triggering barrier formation. Though many factors determine the strength of the ETG drive, such as $\tau$, $\hat{s}$ strongly controls the saturated level of turbulent flux. Local nonlinear simulations show that $\hat{s}$ helps determine the value of the nonlinear critical gradient for significant turbulent transport, which can be much larger than the linear critical gradient and consistent with observations of e-ITB gradient lengths.

Global simulations, which incorporate the experimental radial variations of $\tau, q$, and $\hat{s}$, are able to reproduce the low values of turbulence necessary to allow very steep temperature gradients in the transport barrier. In the outer part of the barrier, where the magnetic shear is not as negative, the ETG-driven flux in the simulation is comparable to the experimentallyinferred heat flux. In the inner part of the transport barrier, where $\tau$ is larger and $\hat{s}$ is even more negative, the ETG-driven turbulence appears to be completely suppressed, and some other mechanism is presumably responsible for the residual transport observed in that part of the barrier. Additionally, the electron flux does not peak at the location of peak linear ETG drive, consistent with the existence of a nonlinear critical gradient for transport that depends at least in part on magnetic shear. This is also consistent with the experimental observation $^{17}$ that peak values of $R / L_{T_{e}}$ in e-ITBs are more strongly correlated with $\hat{s}_{\text {min }}$ than $q_{\min }$.

Though these simulations show that the ETG mode can potentially drive enough turbulent thermal flux to account for experimentally inferred levels, it is possible to suppress ETG-driven turbulence with magnetic shear, thereby allowing the plasma to sustain very steep gradients that are well above the linear threshold for instability. The implications of this effect may very well translate beyond NSTX; as stellarators' external coils can be used to produce negative magnetic shear, this might be a way to reduce turbulence. Other tokamaks may also benefit from current profile control. In short, magnetic shear reversal, even without background flow shear, could allow magnetic fusion devices to sustain electron temperatures gradients that push past the limits imposed by the onset of linear instability. 


\section{ACKNOWLEDGMENTS}

The authors gratefully acknowledge F. Jenko and D. Told for useful discussions of impurity concentrations during this discharge.

This work was performed under the auspices of the U.S. Department of Energy by Lawrence Livermore National Laboratory under Contract DE-AC52-07NA27344 and the Princeton Plasma Physics Laboratory under Contract DE-AC02-09CH11466. This work was supported by the SciDAC Center for the Study of Plasma Microturbulence and used the computational resources of both the Oak Ridge Leadership Computing Facility, located in the National Center for Computational Sciences at Oak Ridge National Laboratory, which is supported by the Office of Science of the Department of Energy under Contract DEAC05-00OR22725, and the National Energy Research Scientific Computing Center, which is supported by the Office of Science under Contract DE-AC02-05CH11231.

\section{REFERENCES}

${ }^{1}$ M. Ono, S. Kaye, Y. Peng, G. Barnes, W. Blanchard, M. Carter, J. Chrzanowski, L. Dudek, R. Ewig, D. Gates, R. Hatcher, T. Jarboe, S. Jardin, D. Johnson, R. Kaita, M. Kalish, C. Kessel, H. Kugel, R. Maingi, R. Majeski, J. Manickam, B. McCormack, J. Menard, D. Mueller, B. Nelson, B. Nelson, C. Neumeyer, G. Oliaro, F. Paoletti, R. Parsells, E. Perry, N. Pomphrey, S. Ramakrishnan, R. Raman, G. Rewoldt, J. Robinson, A. Roquemore,

P. Ryan, S. Sabbagh, D. Swain, E. Synakowski, M. Viola, M. Williams, J. Wilson, and the NSTX Team, Nuclear Fusion 40, 557 (2000).

${ }^{2}$ S. M. Kaye, R. E. Bell, D. Gates, B. P. LeBlanc, F. M. Levinton, J. E. Menard, D. Mueller, G. Rewoldt, S. A. Sabbagh, W. Wang, and H. Yuh, Physical Review Letters 98, 175002 (2007).

${ }^{3}$ S. Kaye, F. Levinton, D. Stutman, K. Tritz, H. Yuh, M. Bell, R. Bell, C. Domier,

D. Gates, W. Horton, J. Kim, B. LeBlanc, N. L. Jr, R. Maingi, E. Mazzucato, J. Menard, D. Mikkelsen, D. Mueller, H. Park, G. Rewoldt, S. Sabbagh, D. Smith, and W. Wang, Nuclear Fusion 47, 499 (2007).

${ }^{4}$ F. Jenko, W. Dorland, and G. W. Hammett, Physics of Plasmas 8, 4096 (2001).

${ }^{5}$ F. Jenko, W. Dorland, M. Kotschenreuther, and B. N. Rogers, Physics of Plasmas 7, 1904 
(2000).

${ }^{6}$ W. Dorland, F. Jenko, M. Kotschenreuther, and B. N. Rogers, Physical Review Letters $85,5579(2000)$.

${ }^{7}$ F. Jenko and W. Dorland, Physical Review Letters 89, 225001 (2002).

${ }^{8}$ W. M. Nevins, J. Candy, S. Cowley, T. Dannert, A. Dimits, W. Dorland, C. Estrada-Mila, G. W. Hammett, F. Jenko, M. J. Pueschel, and D. E. Shumaker, Physics of Plasmas 13, 122306 (2006).

${ }^{9}$ W. M. Nevins, S. E. Parker, Y. Chen, J. Candy, A. Dimits, W. Dorland, G. W. Hammett, and F. Jenko, Physics of Plasmas 14, 084501 (2007).

${ }^{10}$ C. M. Roach, I. G. Abel, R. J. Akers, W. Arter, et al., Plasma Physics and Controlled Fusion 51, 124020 (2009).

${ }^{11}$ J. Candy, R. E. Waltz, M. R. Fahey, and C. Holland, Journal of Physics: Conference Series 78, 012008 (2007).

${ }^{12}$ J. Candy, R. E. Waltz, M. R. Fahey, and C. Holland, Plasma Physics and Controlled Fusion 49, 1209 (2007).

${ }^{13}$ T. Görler and F. Jenko, Physical Review Letters 100, 185002 (2008).

${ }^{14}$ D. R. Smith, E. Mazzucato, W. Lee, H. K. Park, C. W. Domier, and J. Luhmann, Review of Scientific Instruments 79, 123501 (2008).

${ }^{15}$ E. Mazzucato, D. R. Smith, R. E. Bell, S. M. Kaye, J. C. Hosea, B. P. LeBlanc, J. R. Wilson, P. M. Ryan, C. W. Domier, J. Luhmann, H. Yuh, W. Lee, and H. Park, Physical Review Letters 101, 075001 (2008).

${ }^{16}$ E. Mazzucato, R. Bell, S. Ethier, J. Hosea, S. Kaye, B. LeBlanc, W. Lee, P. Ryan, D. Smith, W. Wang, J. Wilson, and H. Yuh, Nuclear Fusion 49, 055001 (2009).

${ }^{17}$ H. Y. Yuh, F. M. Levinton, R. E. Bell, J. C. Hosea, S. M. Kaye, B. P. LeBlanc, E. Mazzucato, J. L. Peterson, D. R. Smith, J. Candy, R. E. Waltz, C. W. Domier, N. C. Luhmann, W. Lee, and H. K. Park, Physics of Plasmas 16, 056120 (2009).

${ }^{18}$ H. Y. Yuh, S. M. Kaye, F. M. Levinton, E. Mazzucato, D. R. Mikkelsen, D. R. Smith, R. E. Bell, J. C. Hosea, B. P. LeBlanc, J. L. Peterson, H. K. Park, and W. Lee, Physical Review Letters 106, 055003 (2011).

${ }^{19}$ R. L. Miller, M. S. Chu, J. M. Greene, Y. R. Lin-Liu, and R. E. Waltz, Physics of Plasmas 5, $973(1998)$.

${ }^{20}$ J. Candy and R. E. Waltz, Journal of Computational Physics 186, 545 (2003). 
${ }^{21}$ J. Candy and E. A. Belli, "GYRO technical guide," General Atomics Report GA-A26818 (General Atomics, 2010).

${ }^{22}$ J. L. Peterson, Ph.D. thesis, Princeton University (2011).

${ }^{23}$ E. A. Belli and J. Candy, Physics of Plasmas 17, 112314 (2010).

${ }^{24}$ A. M. Dimits, G. Bateman, M. A. Beer, B. I. Cohen, W. Dorland, G. W. Hammett, C. Kim, J. E. Kinsey, M. Kotschenreuther, A. H. Kritz, L. L. Lao, J. Mandrekas, W. M. Nevins, S. E. Parker, A. J. Redd, D. E. Shumaker, R. Sydora, and J. Weiland, Physics of Plasmas 7, 969 (2000).

${ }^{25}$ D. R. Mikkelsen and W. Dorland, Physical Review Letters 101, 135003 (2008).

${ }^{26}$ E. A. Belli, G. W. Hammett, and W. Dorland, Physics of Plasmas 15, 092303 (2008).

${ }^{27}$ T. M. Antonsen, J. F. Drake, P. N. Guzdar, A. B. Hassam, Y. T. Lau, C. S. Liu, and S. V. Novakovskii, Physics of Plasmas 3, 2221 (1996).

${ }^{28}$ W. Guttenfelder and J. Candy, Physics of Plasmas 18, 022506 (2011). 